\title{
Management of Chronic Rhinosinusitis with Nasal Polyposis in the Era of Biologics
}

\section{Justin C Morse \\ Craig Miller \\ Brent Senior}

University of North Carolina Department of Otolaryngology-Head and Neck Surgery, University of North Carolina Medical Center, Chapel Hill, NC, 27599, USA
Correspondence: Brent Senior University of North Carolina Department of Otolaryngology-Head and Neck Surgery, University of North Carolina Medical Center, Chapel Hill, NC, 27599, USA

Email Brent.Senior@med.unc.edu
Purpose of Review: Chronic rhinosinusitis with nasal polyposis (CRSwNP) is a phenotypic designation of the broader condition of chronic rhinosinusitis. The advent of targeted biologics has shown promise in targeting different aspects of the inflammatory pathway, yet there remains a lack of consensus on the correct timing and use of these medications. This review seeks to provide a concise update of the available literature on the pathophysiology of CRSwNP, the evolution and cost utility of biologics as it pertains to management of patients with CRSwNP, and evidence for each available biologic and its use in CRSwNP.

Recent Findings: There are two biologics with FDA approval for use in CRSwNP: dupilumab and omalizumab. Recent clinical trials of other biologic therapies targeting type 2 inflammatory pathways have also demonstrated efficacy both in symptom scores and nasal polyp reduction. However, studies have questioned the cost utility of these medications compared to other interventions. Furthermore, timing of use with respect to other interventions including surgery remains challenging.

Keywords: chronic rhinosinusitis with nasal polyposis, biologics, nasal polyps, management of nasal polyps

\section{Summary}

Biologics have demonstrated utility in the management of CRSwNP, yet there is a lack of evidence on durability with cessation of use. The cost of biologics is high with some evidence suggesting less cost utility when compared to surgery. A price reduction would likely improve adoption. While biologics target endotypes within CRSwNP, clinicians remain without the ability to easily identify these different endotypes in CRSwNP patients. As such, biologics are best reserved for refractory cases and use in a postoperative setting in CRSwNP patients. Preoperative consideration for those subtypes of CRSwNP with known refractory disease, like aspirin exacerbated respiratory disease (AERD) and allergic fungal sinusitis (AFS), can be considered but these medications should not be considered substitutes for surgery. Current evidence suggests dupilumab has the most robust evidence for use in the Caucasian, western population with CRSwNP.

\section{Introduction}

Chronic rhinosinusitis with nasal polyposis (CRSwNP) is a phenotypic designation of the broader condition of chronic rhinosinusitis. The epidemiology of CRSwNP has an estimated prevalence of $2-4 \%$ based on geographic region surveyed. ${ }^{1,2}$ It is 
characterized grossly by grape-like projections of inflammatory polyps within the nasal cavity. Diagnosis is made based on chronology of symptomatology and objective evidence on endoscopy or computed tomography imaging demonstrating evidence of polyposis. ${ }^{3}$ CRSwNP is known to cause a significant burden to quality of life, ${ }^{4}$ result in significant health care expenditures, ${ }^{1}$ and more recently has been demonstrated to have an impact on cognitive function. ${ }^{5-8}$ While the exact pathophysiologic mechanisms are yet to be elucidated, evidence evaluating Caucasian populations suggest an predominately type 2 inflammatory reaction and aberrant eosinophilic activation. ${ }^{9}$ However, recent evidence has demonstrated that other mechanisms for development also exist and different inflammatory endotypes exist within this broad phenotypic category and may in part be related to geography. ${ }^{10,11}$

As such recent research has sought to improve classification of this broad phenotypic so that more specific treatment paradigms can be developed targeting specific inflammatory endotypes. ${ }^{12-14}$ Current management strategies employ a combination both of medical and surgical interventions to alleviate disease burden and provide symptom control. Medical therapies target generalized inflammation and include, but are not limited to, steroid nasal sprays, oral steroids, saline rinses, and antibiotics. ${ }^{3}$ The advent of novel targeted biologics have shown promise in targeting a different aspect of the inflammatory pathway that characterizes the majority of western CRSwNP populations, yet there remains a lack of consensus on the correct timing and use of these medications. In particular the role of these medications and timing of surgical intervention remains unanswered. This review seeks to provide a concise update of the available literature on (1) the pathophysiology of CRSwNP, (2) the evolution and cost utility of biologics as it pertains to management of patients with CRSwNP, and (3) evidence for each available biologic and its use in CRSwNP.

\section{Pathophysiology of Chronic Rhinosinusitis with Nasal Polyposis and Current Management}

An understanding of the pathophysiology of CRSwNP is critical to comprehending the role of biologics in management. The 2021 International Consensus Statement on Allergy and Rhinology (ICAR:RS 2021) designates three main types of inflammation involved in CRSwNP with the dominant type being type 2 in Caucasian populations with
CRSwNP characterized by increased IL-5, 4, 13, eosinophilic cationic protein, and p-glycoprotein. ${ }^{3}$ This inflammatory profile has been substantiated in the literature with nasal polyp biopsies in CRS demonstrating elevated levels of innate lymphoid cells (ILC2), macrophages, and mast cells. In contradistinction, Asian populations with CRSwNP have demonstrated a greater proportion of patients with an inflammatory profile mediated by a predominant Th1 and/or Th17 cells. ${ }^{15}$ Multiple studies have demonstrated heterogeneity in endotypes among CRSwNP patients. ${ }^{12,14,16}$

While the exact mechanism for development of polyps remains elusive, current evidence suggests a complex dysregulation of the interface of innate and adaptive immunity. The nasal mucosa shows histologic remodeling that is characterized by epithelial-mesenchymal transition with goblet cell hyperplasia and fibrin deposition. ${ }^{17}$ Other research has also shown contribution from weaknesses in the sinonasal epithelial barrier. Injured epithelium from infection, proteases, and irritants leads to increased production of Th2-promoting cytokines including thymic stromal lymphopoietin (TSLP). ${ }^{18}$ TSLP appears stimulate type 2 ILC2s and mast cells and the release of type 2 cytokines. ${ }^{19}$ Furthermore, in vitro data suggests that the type 2 cytokines IL-4 and IL-13 can result in epithelial barrier dysfunction via increased expression of TSLP. ${ }^{20}$ In summary, patients with CRSwNP have a complex pathophysiologic mechanism that demonstrates heterogeneity between patients. There is multifactorial dysfunction between host and environment at the level of the sinonasal mucosa and this results in immune dysfunction and ultimately persistent inflammation manifesting as the phenotype of polyposis.

Management of these patients can be challenging. Grade A evidence for management of CRSwNP includes use of nasal saline irrigations for maintenance therapy and macrolide antibiotics and systemic steroids for acute exacerbations. ${ }^{3,21}$ Failure of medical management is estimated at $50 \%$ for $\mathrm{CRSwNP}^{22}$ With failure of medical management, surgical intervention is indicated and includes functional endoscopic sinus surgery. Surgical therapy functions to remove polyposis and inflammatory tissue and improve topical delivery of medications. It is important to note surgical intervention is not considered curative but rather a means to improve medical management. Specific subtypes of CRSwNP including aspirin exacerbated respiratory disease and allergic fungal rhinosinusitis have both demonstrated increased refractory 
nature to treatment. ${ }^{16,23,24}$ Many of these patients have concurrent pulmonary disease including asthma. ${ }^{25}$ One benefit of novel biologic agents is that they are able to target both pulmonary and sinonasal inflammation which have been shown to have high correlation. ${ }^{26}$

\section{History and Cost Efficacy of Biologics for Nasal Polyposis}

As previously discussed the aims of treating patients with CRSwNP are to increase quality of life, control disease progression, and to minimize therapeutic side effects. The evolution of biologics in treatment of CRSwNP originated with the treatment of asthma. The united upper airway hypothesis has demonstrated similar inflammatory profiles existing throughout the upper aerodigestive tract which has driven this treatment paradigm. ${ }^{27}$ The first biologic approved for asthma was omalizumab in 2003 and the first to be approved for CRSwNP was dupilumab in 2019. Dupilumab approval was based on a combination of two concurrently run, multi-institutional, randomized control trials titled LIBERTY NP SINUS-24 and LIBERTY NP SINUS-52. ${ }^{28}$ More recently, omalizumab has obtained FDA approval for use in CRSwNP based on concurrently run Phase 3 randomized controlled trials entitled POLYP-1 and POLYP-2. ${ }^{29}$ No current head-tohead trials have been performed evaluating comparative efficacy in CRSwNP.

One of the greatest criticisms of biologic therapy for CRSwNP is cost, particularly in a setting of everincreasing health-care costs. Estimated cost per year remains $>\$ 30,000$ on average. ${ }^{30}$ Cost applied to value of care is traditionally quantified using a quality-adjusted life year (QALY). A QALY is a year of life that has utility quantified between 0 (death) and 1 (perfect health). The benefit of a therapy can be measured in the number of QALYs that are added to a patient's life. ${ }^{31}$ Application of these concepts was performed by Rudmik et al in a 2015 analysis using a Markov decision tree model estimating the value that FESS added to medical treatment of CRS. ${ }^{32}$ This study demonstrated that the addition of FESS resulted in an incremental cost-effectiveness ratio (ICER) of \$5901.90 per QALY leading the authors to conclude that addition of FESS to medical management of CRS was a cost-effective strategy. ${ }^{32}$ A second cost analysis by Scangas et al in 2017, separated patients with CRSwNP and CRSsNP. ${ }^{33}$ This study compared the costs of FESS between these patient populations and found similar results. Most specifically CRSwNP found that the addition of FESS resulted in ICER of $\$ 5687.41$ per QALY. ${ }^{33}$ A single study has performed a cost utility analysis of Dupixent $^{\circledR}$ (dupilumab) vs endoscopic sinus surgery. Scangas et al recently reported a surgical approach to treatment of CRSwNP cost $\$ 50,436.99$ and produced 9.80 QALYs, whereas dupilumab treatment cost $\$ 536,420.22$ and produced only 8.95 QALYs. The authors concluded that endoscopic sinus surgery was both less costly and more effective than dupilumab treatment. Furthermore in a one-way sensitivity analysis, endoscopic sinus surgery was demonstrated to be more cost-effective compared to dupilumab regardless of the frequency of revision surgery and at any yearly cost of dupilumab above $\$ 855 .^{30}$ We can further extrapolate the cost effectiveness based on the value of biologics in treatment of asthma. Cost effectiveness studies have universally recommended that pharmaceutical companies decrease their prices by $60-80 \%$ to make their drugs more costeffective as the maximum cost efficiency was only found in patients with poorly controlled disease. ${ }^{34-36}$

Further research into the cost effectiveness of these therapies will be critical to understanding their utility particularly in refractory cases and use as adjuvant therapies. At present the current data suggests lower cost effectiveness compared to surgical intervention. It should be noted that true cost is additionally likely underestimated due to the less accurate prediction of future costs given that the novelty of these drugs precludes knowledge of their longterm side effects. ${ }^{31}$

\section{Anti-IgE}

Omalizumab in an anti-IgE monoclonal antibody that binds to the Fc receptor on a multitude of different inflammatory cells including mast cells and basophils. It functions to reduce total serum levels of $\operatorname{IgE}$ and downstream effects of IgE mediated release of inflammatory cytokines. It is marketed under the trade name of Xolair and was originally FDA approved for use for the treatment of moderate to severe persistent allergic asthma in people 6 years of age or older whose asthma symptoms are not controlled by inhaled corticosteroids, and for chronic idiopathic urticaria in people 12 years of age and older. ${ }^{37,38}$ It is given via a subcutaneous injection every 2-4 weeks with dose determined by serum total $\mathrm{IgE}$ and body weight. Its role in CRSwNP was based on literature suggesting a pathophysiologic mechanism related to local intranasal $\operatorname{IgE}$ production that leads to an inflammatory cascade. 
Moreover, specific IgE antibodies have been identified in nasal polyp tissue independently of their presence in serum suggesting localized effect. ${ }^{39}$

Further clinical evidence for its efficacy in CRSwNP was originally assessed in a double blinded placebocontrolled trial by Gevaert et al and demonstrated a reduction in total endoscopic nasal polyp score (NPS), improved pre and post treatment computed tomography (CT) scores, improved nasal peak inspiratory flow, as well as symptoms related to allergy and asthma quality of life measures. No change in smell function was found. ${ }^{40}$ A clinical trial by Pinto et al demonstrated contradictory results without statistically significant changes in any of the aforementioned measurements. ${ }^{41}$ This was further confirmed in a meta-analysis by Rivero et al showed a decrease in NPS only in patients with concurrent asthma. ${ }^{42}$ More recent evidence from Phase III clinical trials, Polyps I and II has demonstrated efficacy in treatment of CRSwNP and in December of 2020 FDA granted approval for Omalizumab for treatment of adults with refractory CRSwNP. ${ }^{29}$ These trials similarly looked at NPS, nasal congestion scores (NCS), and quality of life measures measured by SNOT-22. While both randomized placebo-controlled trials demonstrated reduction of polyp scores compared to placebo, nasal congestion scores, and improved SNOT-22 scores, the clinically meaningful difference of polyp reduction and NCS remains difficult to assess. Further confounders of designation of proportion of allergic patients in each cohort was not reported. While SNOT-22 scores again demonstrated clinically meaningful reduction, it remains unclear if these are lasting in the long term and the role surgical intervention could play in this treatment management.

A single study has compared use omalizumab to surgical intervention. This study specifically compared patients with severe allergic asthma and concurrent CRSwNP receiving omalizumab and those treated with endonasal endoscopic surgery which similarly demonstrated reductions in SNOT22 scores at 16 weeks of treatment. This study was limited to only severe allergic asthma patients with concurrent polyp disease and lacked long term follow up. Furthermore, the role of omalizumab as an adjuvant to surgical intervention has yet to be elucidated. ${ }^{43}$ Further cost-effective studies are needed to justify its use in these settings.

\section{Anti-II-5}

IL-5 is a key cytokine released in the Th- 2 inflammatory cascade. Release, in conjunction with IL-4 and IL-13, drives increased local production of $\operatorname{IgE}$ and results in eosinophilia, chemotaxis, differentiation, and activation of eosinophil survival. ${ }^{44}$ Due to its central role in eosinophil activity, prevention of IL-5 binding to the IL-5R (IL5Ra) subunit on the eosinophil surface has been targeted as a therapeutic strategy, especially in light of the ubiquitous nature of IL-5 in nasal polyps (present in $80 \%$ of nasal polyps in certain populations). IL-5 is the critical factor that promotes eosinophil development and survival and therefore blockade also exhibits secondary effects via elimination of eosinophils peripherally and within tissue. IL-5 also appears to have a key role in the pathogenesis of nasal polyposis including expression of IL-5 in nasal polyps, this expression associated with asthma comorbidity, and eosinophilic inflammation associated with polyp recurrence after surgery. ${ }^{45}$ A number of monoclonal antibodies have been developed and investigated.

Mepolizumab (Nucala) is an anti-IL-5 monoclonal antibody currently under investigation as a potential therapy for nasal polyposis. Mepolizumab has been shown to reduce blood and tissue eosinophil counts and is currently approved for treatment of severe eosinophilic asthma. ${ }^{46,47}$ Bachert et al performed a randomized trial investigating the use of Mepolizumab in patients with nasal polyposis. Patients with severe recurrent nasal polyposis who required surgery were randomized to $750 \mathrm{mg}$ of mepolizumab or placebo every 4 weeks via IV infusion for 6 doses. All patients received intranasal corticosteroids for the course of the study. The primary aim of the study was patients no longer meeting requirement for surgery 4 weeks after final dose based on endoscopic nasal polyp scores and nasal polyposis severity VAS scores. Secondary endpoints included nasal polyp scores throughout the study period, pulmonary function, and olfactory function. Results showed a decreased need for surgery and improvement in NPS and nasal polyp VAS scores in the treatment group. Mepolizumab also improved olfactory function compared to placebo. Treatment led to a 10 -fold reduction in eosinophil counts at week 25 , which mirrored improvement in symptoms and need for surgery. Interestingly, there was no change in PFT parameters despite improvement in eosinophilia. This study did not follow patients after discontinuation of the drug and therefore conclusions about the durability and long-term efficacy cannot be made. The short study duration, low number of patients, lack of comorbid severe eosinophilic asthma, criteria for surgical intervention, and high drop-out rate of study participants reduce its clinical generalizability. ${ }^{48}$ Nonetheless, 
mepolizumab improved nasal polyp scores, symptoms of nasal polyposis, and possibly need for surgery compared to placebo. The more recent SYNAPSE study conducted by Taberrer et al enrolled 407 adults with CRSwNP for a 52 -week study using $100 \mathrm{mg}$ subcutaneously administered mepolizumab. Again, mepolizumab offered clinically and statistically significant improvements in NPS and symptom scores compared to placebo. ${ }^{49}$

Benralizumab (Fasenra) is a afucosylated monoclonal antibody that directly targets the alpha chain of the IL-5 receptor and has demonstrated strong apoptotic effects on eosinophils. This is in contrast to mepolizumab method of action of binding to IL-5 protein in circulation. Benralizumab binding to the IL-5 receptor on the surface of eosinophils leads to degradation of signaling, antibodydependent cell mediated cytotoxicity, and apoptosis. ${ }^{50}$ Like other IL-5 targeting therapies, benralizumab has been shown to lead to reduction in proinflammatory processes in patients with asthma, but until recently had not been shown to be effective for nasal polyposis. ${ }^{51,52}$

Tversky et al recently performed a randomized, double-blind placebo-controlled study on benralizumab $30 \mathrm{mg}$ delivered subcutaneously over 20 weeks in patients with severe surgically-recalcitrant, bilateral CRSwNP, high peripheral eosinophilia, and recent use of oral corticosteroids. Primary outcome measure was NPS. Changes in imaging scores (Lund-MacKay), the Sino-Nasal Outcome Test (SNOT-22) scores, and nasal blockage scores, as well as olfaction measures served as secondary endpoints. 24 patients were randomized. While there was an overall greater improvement in nasal polyp score and LundMacKay scores in the benralizumab treated group there was wide variation in degree of improvement within the benralizumab group with some patients showing significant improvement and many showing minimal to no improvement. Nonetheless, the authors conclude that benralizumab significantly reduced nasal polyp size and nasal blockage score and increased olfaction compared to baseline. Of note, there was improvement in many of the outcome measures in the placebo group in the study and this was attributed to use of rescue intranasal and systemic corticosteroids during the study period. ${ }^{53}$

\section{Anti-II-4/I 3}

Dupilumab (Dupixent ${ }^{\circledR}$ ) is an anti-IL-4 monoclonal antibody which functions by targeting the alpha chain of the IL-4Ra, the common receptor for both IL-4 and IL-13. Both IL-4 and IL-13 play a central role in the Th2 pathway and ultimately polyp formation. Dupilumab has been approved to treat severe atopic dermatitis and has shown efficacy in treating asthma. In 2019, it was the first biologic approved by the FDA for the treatment of CRSwNP. The impetus for approval was based on two randomized, placebo-controlled trials, the LIBERTY-24 and LIBERTY52 studies. ${ }^{28}$ Patients with CRSwNP, with or without concomitant asthma, refractory to intranasal corticosteroids and who had received systemic corticosteroids or had previous sinonasal surgery in the preceding two years were randomized into treatment with subcutaneous dupilumab $300 \mathrm{mg}$ vs placebo. In the 24-week study, patients received their dose every other week, while in the 52-week study patients initially received their dose every two weeks then at the 24 week mark they were randomized to receive either two or four-week dosing. Primary endpoints in both studies were change in baseline NPS and nasal congestion severity. Secondary endpoints including Lund-MacKay CT score, total symptom score, olfactory function, SNOT-22 score, and pulmonary function. Patients in both studies showed improvement in all measures during the treatment course both from baseline and compared to placebo. The fragility of these improvements was highlighted by following patients in the 24-week study, which showed recurrence of polyps and worsening nasal obstruction after cessation of the therapy. Dupilumab greatly reduced the need for systemic steroids, improved symptoms, including olfaction, and health-related quality of life. Dupilumab treatment also reduced type 2 biomarkers in serum and nasal secretions, including total $\operatorname{IgE}$. The lack of follow-up of patients after the 52-week trial calls into question durability of the therapy following cessation. ${ }^{28}$ Based on the results of this trial, dupilumab was approved for use in patients with nasal polyposis.

\section{Other Biologics Under Trial}

Further research into the pathophysiologic mechanisms in CRSwNP will likely yield future biologic targets in these disease processes. At present several new agents are under investigation. One of the most exciting prospects is antiTSLP antibodies. As previously discussed TSLP is critical factor in the inflammatory pathway that has been hypothesized to contribute to polyp formation. Its investigation was initially for allergen-induced asthma. Initial trial responses showed that treatment with an anti-TSLP antibody lowered sputum eosinophil counts and FeNO levels and reduced allergen-induced bronchoconstriction. ${ }^{54}$ A separate Phase 2 study demonstrated reduction in 
asthma exacerbations and mild increase in pulmonary function with use of the anti-TSLP antibody tezepelumab. ${ }^{55}$ More recent work in CRSwNP has shown increased expression and/or activity of TSLP in nasal polyp tissue compared to healthy sinus tissue or that from patients with CRSsNP. ${ }^{56}$ This is would suggest this target may be of significant value in treatment of CRSwNP.

Another inflammatory mediator target with potential utility is anti-IL-33 antibodies. IL-33 is known to play a role in the pathogenesis of allergic disease and as a neutrophil activator. ${ }^{57}$ In addition to its likely role in allergic asthma, increased expression of IL-33 has been found in the sinus tissue of patients with CRSwNP. ${ }^{58}$ More specifically elevated levels have been demonstrated in AERD patient polyps, this may play a specific role in future treatment of these patients. ${ }^{59}$ Several anti-IL-33 biologics have been developed and preliminarily evaluated in asthmatic patients (NCT03469934, NCT03112577) with favorable outcomes. Recent completion of trial of etokimab in CRSwNP patients did not demonstrate statistical differences in symptom scores or NPS (NCT03614923). Further trials are ongoing for the application of this class of agents for the treatment of CRSwNP and will be necessary to elucidate its use.

\section{Discussion}

Biologic medications clearly add value to the future treatment algorithm of CRSwNP. While the classic phenotypic classification of CRSwNP is convenient, current evidence demonstrating the complexities of this disease demonstrate that a phenotypic classification does not adequately address the complexities and differences among patients with this disease process. Current treatment paradigms recommend initial management of a nasal regimen including nasal steroids, saline nasal rinses, intranasal antihistamines with oral steroids and antibiotics utilized for exacerbations. ${ }^{3}$ These medications have broad effect on the majority of different types of inflammation and should continue to be used as the mainstay of management in these diseases. However, due to high failure rates of medical management alone, surgery continues to play a clear role in improving medical management and quality of life. ${ }^{60}$ Current research does not suggest equivalency among biologics and surgical intervention. As such we the authors feel that use of a biologic should be as an adjuvant to surgical therapy rather than in place or to avoid it. Yet, no consensus exists on the correct timing and use of biologics in these patients. The authors would argue that biologics are best utilized for refractory disease in a postoperative setting. We predicate this argument on the fact that no biologic study has demonstrated a reduction in polyp score to 0 . Surgical intervention theoretically will lead to an immediate postoperative NPS of 0. It is well known that quality of life after FESS results in long lasting and significant real reduction in SNOT-22 scores in CRSwNP. ${ }^{61,62}$ While challenging to directly compare to biologic studies, the durability of surgical intervention does appear to be improved compared to dupilumab. ${ }^{28,61}$ The substantial cost to biologic medications would further suggest these should be utilized when other alternatives fail particularly when QALY is directly compared to surgical interventions. ${ }^{30,31}$ Despite good surgical intervention and medical management, a subset of patients will demonstrate refractory disease. We would argue this is an optimum time for utilization of biologics. Furthermore, patients known to demonstrate refractory disease (ie those needing substantial numbers of revision surgeries) should be considered for adjuvant biologic therapy postoperatively. Patients with aspirin exacerbated respiratory disease (AERD) and allergic fungal sinusitis (AFS) which are phenotypic representations of more refractory disease are one example of patients in whom a higher consideration for utilization of a biologic for maintenance of disease control should be strongly considered. ${ }^{26}$ The authors' suggested algorithm for use of biologic therapy is displayed in Figure 1 with a summary of available biologics in Table 1.

Without a clear means to evaluating a patient's inflammatory endotype at this time, use of these medications remains predicated on the generalized phenotypic evaluation. We feel that surgical intervention provides histopathologic clues to inflammatory subtypes for which biologics can be used, ie eosinophilic infiltrate or neutrophilic infiltrate within the polyp tissue. The question to starting a biologic in the pre- or postoperative setting remains challenging with limited evidence to support one treatment strategy over another. The evidence to suggest that biologics are preventative of future surgical intervention is sparse at best. While Bachert et al suggested lower need for surgical intervention with use of mepolizumab, the short study time period does not give a clear picture to the reality of reduction in need for surgery as the majority of polyp patients do very well postoperatively for $>1$ year and determination of surgical candidacy was based on endoscopic NPS and VAS. ${ }^{48}$ Future evaluation and trials 


\section{Chronic Rhinosinusitis with Nasal Polyps Patient \\ I \\ Initiate Appropriate Medical Management<smiles>[AlH2]</smiles> \\ Refractory/Persistent Symptoms? \\ I ves \\ Co existing disease with indication for biologic (i.e. eosinophilic asthma; eczema), prior surgery, or other indication of recalcitrant disease (AERD,AFS, multiple revision surgeries) ? \\ Yes

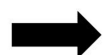 \\ Sufficient ESS (if surgical candidate) + Consideration of Biologic \\ $\checkmark N o$ \\ Sufficient ESS \\ I \\ Improvement in Symptoms

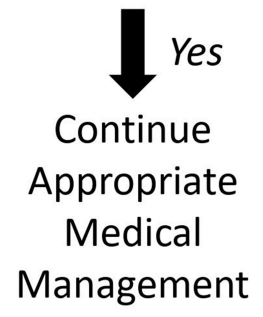 \\ $\coprod^{\text {No }}$ \\ Consideration \\ of Biologic}

Figure I Algorithm proposed by authors for use of biologics in the management of chronic rhinosinusitis with nasal polyps.

Abbreviations: ESS, endoscopic sinus surgery; AERD, aspirin exacerbated respiratory disease; AFS, allergic fungal sinusitis.

of combination modality treatment will be necessary to improve our understanding of biologics effect on the need for surgery.

Further, challenging the use of management of these medications is that there are no current head-to-head trials of using different biologics. This raises the question to the practitioner as to which biologic to use for which patient. This can be particularly perplexing when the clinical context of each patient does not provide a secondary diagnosis that would be responsive to a particular biologic (ie allergic asthma as a candidate for omalizumab or eosinophilic asthma for mepolizumab). Current FDA approval and trial data would suggest dupilumab as the most efficacious choice in Caucasian populations with CRSwNP in these situations. This may be secondary to its theoretical effect on a more upstream mediator of type 2 inflammation. Without the ability to easily identify endotypes at present we, as clinicians, are left to extrapolate clinical signs and symptoms that suggest one endotype over another. We however do not recommend using biologics on all CRSwNP patients as the cost does not appear to justify the efficacy over other treatment strategies. While the use of serum markers (ie total IgE or serum eosinophils) as a means to pseudo-endotyping CRSwNP patients is commonly practiced and based on prior asthma trials, the data to support this clinical practice in CRSwNP is conflicting and with evidence demonstrating lack of direct correlation. ${ }^{63}$ Histopathologic analysis of polyp tissue continues to demonstrate highest correlation with different types of inflammation and currently is the best option for helping to make this determination, ${ }^{64}$ but even it has limited diagnostic utility given that multiple 
Table I Table Describing Biologics Discussed Including: Name, Target, FDA Approvals, Mechanism, Dosing and Side Effects

\begin{tabular}{|c|c|c|c|c|c|}
\hline $\begin{array}{l}\text { Generic } \\
\text { Name }\end{array}$ & $\begin{array}{l}\text { Trade } \\
\text { Name }\end{array}$ & Target & FDA Approvals & Dosing & Side Effects \\
\hline Omalizumab & Xolair & $\begin{array}{l}\text { Anti-lgE via Fc } \\
\text { receptor blockade }\end{array}$ & $\begin{array}{l}\text { CRSwNP; Allergic } \\
\text { Asthma; Chronic } \\
\text { Uticaria }\end{array}$ & $\begin{array}{l}\text { Subcutaneous injection every } 2-4 \\
\text { weeks }\end{array}$ & $\begin{array}{l}\text { - Sinusitis } \\
\text { - Headache } \\
\text { - Pharyngitis } \\
\text { - Injection site reaction } \\
\text { - } 0.2 \% \text { risk of anaphylaxis }\end{array}$ \\
\hline Mepolizumab & Nucala & Anti-IL5 & Eosinophilic Asthma & Monthly subcutaneous injections & $\begin{array}{l}\text { - Headache } \\
\text { - Fatigue } \\
\text { - Injection site reaction }\end{array}$ \\
\hline Benralizumab & Fasenra & $\begin{array}{l}\text { Anti-IL5 via IL5-R } \\
\text { alpha receptor } \\
\text { blockade }\end{array}$ & & $\begin{array}{l}\text { Monthly subcutaneous injections } \\
\text { for } 3 \text { months then every other } \\
\text { month }\end{array}$ & $\begin{array}{l}\text { - Headache } \\
\text { - Pharyngitis } \\
\text { - Injection site reaction }\end{array}$ \\
\hline Dupilumab & Dupixent & $\begin{array}{l}\text { Anti-IL4 and IL-1 } 3 \text { via } \\
\text { IL-4Ra receptor } \\
\text { blockade }\end{array}$ & $\begin{array}{l}\text { CRSwNP; Atopic } \\
\text { Dermatitis }\end{array}$ & $\begin{array}{l}\text { Subcutaneous injection every } \\
\text { other week }\end{array}$ & $\begin{array}{l}\text { - Nasopharyngitis } \\
\text { - Headache } \\
\text { - Injection site reaction }\end{array}$ \\
\hline
\end{tabular}

endotypes within CRSwNP and even subendotypes in AERD have been identified in basic science and clinical research settings with similar histopathologic findings. ${ }^{11,14,16}$ Clearly, the ability to easily identify these different patients remains challenging in an outpatient clinical setting. Ultimately, clinical success will be predicated on the accessibility of easily obtainable biomarker-based endotyping diagnostic tools and patient response analyses. Theoretically this will allow clinicians to precisely match a patient to the appropriate biologic and optimizing an individualized therapeutic strategy. ${ }^{26}$

\section{Conclusion}

Biologic medications have demonstrated utility in the management of CRSwNP with benefits of improving quality of life assessments, reducing polyp scores, and reducing nasal congestion scores, yet there is a lack of durability with cessation of use. Furthermore, the cost of biologics is high with some evidence suggesting less cost utility when compared to surgery and clinicians remain without the ability to easily identify different endotypes in CRSwNP patients basing choice of biologic therapy on best clinical judgement. A price reduction would likely improve adoption into a more systematic medical regimen. These are limitations in adoption of use. We would argue that a majority of patients with CRSwNP can be effectively managed with sufficient surgical intervention and standard medical therapy alone with use of biologics reserved only for refractory cases. Furthermore, we feel surgery provides the greatest means to reducing polyposis and improving quality of life, as such we recommend use of these agents only in the refractory postoperative patient and consideration for use in phenotypic subtypes of CRSwNP with known refractory disease, like AERD and AFS. Current evidence suggests that dupilumab is the choice with the most robust evidence for its use in Caucasian, western populations with CRSwNP. Further head-to-head trials will be necessary to delineate which biologic is best for which patient. In conclusion with further study and price reduction biologics it may prove to be a useful adjuvant medication for patients with recalcitrant CRSwNP.

\section{Disclosure}

Brent Senior is the Vice President for Development and Strategy for the American Rhinologic Society. The authors report no other conflicts of interest in this work.

\section{References}

1. DeConde AS, Soler ZM. Chronic rhinosinusitis: epidemiology and burden of disease. Am $J$ Rhinol Allergy. 2016;30(2):134-139. doi:10.2500/ajra.2016.30.4297

2. Ahn JC, Kim JW, Lee CH, Rhee CS. Prevalence and risk factors of chronic rhinosinusitus, allergic rhinitis, and nasal septal deviation results of the Korean national health and nutrition survey 20082012. JAMA Otolaryngol Head Neck Surg. 2016;142(2):162-167. doi:10.1001/jamaoto.2015.3142

3. Orlandi RR, Kingdom TT, Smith TL, et al. International consensus statement on allergy and rhinology: rhinosinusitis 2021. Int Forum Allergy Rhinol. 2021;11(3):213-739. doi:10.1002/alr.22741

4. Soler ZM, Sauer D, Mace J, Smith TL. Impact of mucosal eosinophilia and nasal polyposis on quality-of-life outcomes after sinus surgery. Otolaryngol Head Neck Surg. 2010;142(1):64-71. doi:10.1016/j. otohns.2009.10.005 
5. Katotomichelakis M, Simopoulos E, Zhang N, et al. Olfactory dysfunction and asthma as risk factors for poor quality of life in upper airway diseases. Am J Rhinol Allergy. 2013;27(4):293-298. doi:10.2500/ajra.2013.27.3903

6. Erskine SE, Hopkins C, Clark A, et al. Chronic rhinosinusitis and mood disturbance. Rhinol J. 2017;55(2):113-119. doi:10.4193/ rhin 16.111

7. Tomoum MO, Klattcromwell C, Delsignore A, Ebert C, Senior BA. Depression and anxiety in chronic rhinosinusitis. Int Forum Allergy Rhinol. 2015;5(8):674-681. doi:10.1002/alr.21528

8. Litvack JR, Mace J, Smith TL. Role of depression in outcomes of endoscopic sinus surgery. Otolaryngol Head Neck Surg. 2011;144 (3):446-451. doi:10.1177/0194599810391625

9. Turner JH, Li P, Chandra RK. Mucus T helper 2 biomarkers predict chronic rhinosinusitis disease severity and prior surgical intervention. Int Forum Allergy Rhinol. 2018;8(10):1175-1183. doi:10.1002/ alr.22160

10. Wen W, Liu W, Zhang L, et al. Increased neutrophilia in nasal polyps reduces the response to oral corticosteroid therapy. J Allergy Clin Immunol. 2012;129(6):1522-1528.e5. doi:10.1016/j.jaci.2012.01.079

11. Turner JH, Chandra RK, Li P, Bonnet KSD. Identification of clinically relevant chronic rhinosinusitis endotypes using cluster analysis of mucus cytokines. J Allergy Clin Immunol. 2018;141(5):1895-7.

12. Chapurin N, Li P, Chandra RK, Turner JH, Chowdhury NI. Elevated mucus interleukin-17A levels are associated with increased prior sinus surgery for chronic rhinosinusitis. Int Forum Allergy Rhinol. 2021;11(2):120-127. doi:10.1002/alr.22652

13. Smith TL, Schlosser RJ, Soler ZM, et al. Olfactory cleft mucus inflammatory proteins in CRS: a Case-Control Study. Int Forum Allergy Rhinol. 2021. doi:10.1002/alr.22770

14. Morse JC, Li P, Ely KA, et al. Chronic rhinosinusitis in elderly patients is associated with an exaggerated neutrophilic proinflammatory response to pathogenic bacteria. $J$ Allergy Clin Immunol. 2019;143(3):990-1002.e6. doi:10.1016/j.jaci.2018.10.056

15. Wang X, Zhang N, Bo M, et al. Diversity of TH cytokine profiles in patients with chronic rhinosinusitis: a Multicenter Study in Europe, Asia, and Oceania. J Allergy Clin Immunol. 2016;138(5):1344-1353. doi:10.1016/j.jaci.2016.05.041

16. Scott WC, Cahill KN, Milne GL, et al. Inflammatory heterogeneity in aspirin-exacerbated respiratory disease. J Allergy Clin Immunol. 2020;147(4):1318-1328.e5. doi:10.1016/j.jaci.2020.11.001

17. Meng J, Zhou P, Liu Y, et al. The development of nasal polyp disease involves early nasal mucosal inflammation and remodelling. PLoS One. 2013;8(12):e82373. doi:10.1371/journal.pone.0082373

18. Schleimer RP. Immunopathogenesis of chronic rhinosinusitis and nasal polyposis. Ann Rev Pathol Mech Dis. 2017;12(1):331-357. doi:10.1146/annurev-pathol-052016-100401

19. Ho J, Bailey M, Zaunders J, et al. Group 2 innate lymphoid cells (ILC2s) are increased in chronic rhinosinusitis with nasal polyps or eosinophilia. Clin Exp Allergy. 2015;45(2):394-403. doi:10.1111/ cea. 12462

20. Nonaka M, Ogihara N, Fukumoto A, et al. Synergistic induction of macrophage inflammatory protein-3 $\alpha /$ CCL2 20 production by interleukin-17A and tumor necrosis factor- $\alpha$ in nasal polyp fibroblasts. World Allergy Organ J. 2009;2(10):218-223. doi:10.1097/wox.0b01 3e3181bdd219

21. Fokkens WJ, Lund VJ, Hopkins C, et al. European position paper on rhinosinusitis and nasal polyps 2020. Rhinology. 2020;58 (SupplS29):1-464. doi:10.4193/Rhin20.600

22. Lam K, Kern RC, Luong A. Is there a future for biologics in the management of chronic rhinosinusitis? Int Forum Allergy Rhinol. 2016;6(9):935-942. doi:10.1002/alr.21780

23. Stevens WW, Schleimer RP. Aspirin-exacerbated respiratory disease as an endotype of chronic rhinosinusitis. Immunol Allergy Clin North Am. 2016;36(4):669-680. doi:10.1016/j.iac.2016.06.004
24. Morse JC, Shilts MH, Ely KA, et al. Patterns of olfactory dysfunction in chronic rhinosinusitis identified by hierarchical cluster analysis and machine learning algorithms. Int Forum Allergy Rhinol. 2019;9 (3):255-264. doi:10.1002/alr.22249

25. Stevens WW, Ocampo CJ, Berdnikovs S, et al. Cytokines in chronic rhinosinusitis role in eosinophilia and aspirin-exacerbated respiratory disease. Am J Respir Crit Care Med. 2015;192(6):682-694. doi:10.1164/rccm.201412-22780C

26. Laidlaw TM, Buchheit KM. Biologics in chronic rhinosinusitis with nasal polyposis. Ann Allergy Asthma Immunol. 2020;124(4):326-332. doi:10.1016/j.anai.2019.12.001

27. Chang CC. Sinusitis, rhinitis, asthma, and the single airway hypothesis. In: Diseases of the Sinuses. New York: Springer;2014:173-194. doi:10.1007/978-1-4939-0265-1 11

28. Bachert C, Han JK, Desrosiers M, et al. Efficacy and safety of dupilumab in patients with severe chronic rhinosinusitis with nasal polyps (LIBERTY NP SINUS-24 and LIBERTY NP SINUS-52): results from two multicentre, randomised, double-blind, placebo-controlled, parallel-group phase 3 trials. Lancet. 2019;394 (10209):1638-1650. doi:10.1016/S0140-6736(19)31881-1

29. Gevaert P, Omachi TA, Corren J, et al. Efficacy and safety of omalizumab in nasal polyposis: 2 randomized phase 3 trials. $J$ Allergy Clin Immunol. 2020;146(3):595-605. doi:10.1016/j. jaci.2020.05.032

30. Scangas GA, Wu AW, Ting JY, et al. Cost utility analysis of dupilumab versus endoscopic sinus surgery for chronic rhinosinusitis with nasal polyps. Laryngoscope. 2021;131(1):E26-E33. doi:10.1002/ lary. 28648

31. Brown WC, Senior B, Critical A. Look at the efficacy and costs of biologic therapy for chronic rhinosinusitis with nasal polyposis. Curr Allergy Asthma Rep. 2020;20(6). doi:10.1007/s11882-020-00910-y

32. Rudmik L, Soler ZM, Mace JC, Schlosser RJ, Smith TL. Economic evaluation of endoscopic sinus surgery versus continued medical therapy for refractory chronic rhinosinusitis. Laryngoscope. 2015;125(1):25-32. doi:10.1002/lary.24916

33. Scangas GA, Remenschneider AK, Su BM, Shrime MG, Metson R. Cost utility analysis of endoscopic sinus surgery for chronic rhinosinusitis with and without nasal polyposis. Laryngoscope. 2017;127 (1):29-37. doi:10.1002/lary.26169

34. Anderson WC, Szefler SJ. Cost-effectiveness and comparative effectiveness of biologic therapy for asthma: to biologic or not to biologic? Ann Allergy Asthma Immunol. 2019;122(4):367-372. doi:10.1016/j.anai.2019.01.018

35. Whittington MD, McQueen RB, Ollendorf DA, et al. Assessing the value of mepolizumab for severe eosinophilic asthma: a cost-effectiveness analysis. Ann Allergy Asthma Immunol. 2017;118 (2):220-225. doi:10.1016/j.anai.2016.10.028

36. Lam J, Hay JW, Salcedo J, Kenyon NJ. A cost-effectiveness analysis of reslizumab in the treatment of poorly controlled eosinophilic asthma. J Asthma. 2019;56(8):872-881. doi:10.1080/02770903.20 18.1500584

37. Hanania NA, Alpan O, Hamilos DL, et al. Omalizumab in severe allergic asthma inadequately controlled with standard therapy: a randomized trial. Ann Intern Med. 2011;154(9):573-582. doi:10.7326/0003-4819-154-9-201105030-00002

38. Doroudchi A, Pathria M, Modena BD. Asthma biologics: comparing trial designs, patient cohorts and study results. Ann Allergy Asthma Immunol. 2020;124(1):44-56. doi:10.1016/j.anai.2019.10.016

39. Zhang N, Holtappels G, Gevaert P, et al. Mucosal tissue polyclonal $\mathrm{IgE}$ is functional in response to allergen and SEB. Allergy Eur J Allergy Clin Immunol. 2011;66(1):141-148. doi:10.1111/j.13989995.2010.02448.x

40. Gevaert P, Calus L, Van Zele T, et al. Omalizumab is effective in allergic and nonallergic patients with nasal polyps and asthma. $J$ Allergy Clin Immunol. 2013;131(1):110-116.e1. doi:10.1016/j. jaci.2012.07.047 
41. Pinto JM, Mehta N, DiTineo M, Wang J, Baroody FM, Naclerio RM. A randomized, double-blind, placebo-controlled trial of anti-IgE for chronic rhinosinusitis. Rhinol J. 2010;48(3):318-324. doi:10.4193/ rhin09.144

42. Rivero A, Liang J. Anti-IgE and anti-IL5 biologic therapy in the treatment of nasal polyposis: a systematic review and meta-analysis. Ann Otol Rhinol Laryngol. 2017;126(11):739-747. doi:10.1177/ 0003489417731782

43. Bidder T, Sahota J, Rennie C, Lund VJ, Robinson DS, Kariyawasam HH. Omalizumab treats chronic rhinosinusitis with nasal polyps and asthma together-a real life study. Rhinol J. 2018;56(1):42-45. doi:10.4193/rhin17.139

44. Kim H, Ellis AK, Fischer D, et al. Asthma biomarkers in the age of biologics. Allergy Asthma Clin Immunol. 2017;13(1). doi:10.1186/ s13223-017-0219-4

45. Van Zele T, Holtappels G, Gevaert P, Bachert C. Differences in initial immunoprofiles between recurrent and nonrecurrent chronic rhinosinusitis with nasal polyps. Am J Rhinol Allergy. 2014;28(3):192-198. doi:10.2500/ajra.2014.28.4033

46. Ortega HG, Liu MC, Pavord ID, et al. Mepolizumab treatment in patients with severe eosinophilic asthma. $N$ Engl J Med. 2014;371 (13):1198-1207. doi:10.1056/nejmoa1403290

47. Flood-Page PT, Menzies-Gow AN, Kay AB, Robinson DS. Eosinophil's role remains uncertain as anti-interleukin-5 only partially depletes numbers in asthmatic airway. Am J Respir Crit Care Med. 2003;167(2):199-204. doi:10.1164/rccm.200208-789OC

48. Bachert C, Sousa AR, Lund VJ, et al. Reduced need for surgery in severe nasal polyposis with mepolizumab: randomized trial. J Allergy Clin Immunol. 2017;140(4):1024-1031.e14. doi:10.1016/j. jaci.2017.05.044

49. Lee S, Tabberer M, Trigg A, et al. Mepolizumab improves health related quality of life for patients with chronic rhinosinusitis with nasal polyps: data from the SYNAPSE Study. J Allergy Clin Immunol. 2021;147(2):AB125. doi:10.1016/j.jaci.2020.12.458

50. Ghazi A, Trikha A, Calhoun WJ. Benralizumab - a humanized mAb to IL-5R $\alpha$ with enhanced antibody-dependent cell-mediated cytotoxicity - a novel approach for the treatment of asthma. Expert Opin Biol Ther. 2012;12(1):113-118. doi:10.1517/14712598.2012.642359

51. Matsuno O, Minamoto S. Rapid effect of benralizumab for severe asthma with chronic rhinosinusitis with nasal polyps. Pulm Pharmacol Ther. 2020;64:101965. doi:10.1016/j.pupt.2020.101965

52. Lombardo N, Pelaia C, Ciriolo M, et al. Real-life effects of benralizumab on allergic chronic rhinosinusitis and nasal polyposis associated with severe asthma. Int $J$ Immunopathol Pharmacol. 2020;34:205873842095085. doi:10.1177/2058738420950851
53. Tversky J, Lane AP, Azar A. Benralizumab effect on severe chronic rhinosinusitis with nasal polyps (CRSwNP): a randomized double-blind placebo-controlled trial. Clin Exp Allergy. 2021;51 (6):836-844. doi:10.1111/cea.13852

54. Gauvreau GM, O'Byrne PM, Boulet L-P, et al. Effects of an anti-TSLP antibody on allergen-induced asthmatic responses. $N$ Engl J Med. 2014;370(22):2102-2110. doi:10.1056/ nejmoa1402895

55. Corren J, Parnes JR, Wang L, et al. Tezepelumab in adults with uncontrolled asthma. $N$ Engl J Med. 2017;377(10):936-946. doi:10.1056/nejmoa1704064

56. Nagarkar DR, Poposki JA, Tan BK, et al. Thymic stromal lymphopoietin activity is increased in nasal polyps of patients with chronic rhinosinusitis. J Allergy Clin Immunol. 2013;132(3):593-600.e12. doi:10.1016/j.jaci.2013.04.005

57. Stolarski B, Kurowska-Stolarska M, Kewin P, Xu D, Liew FY. IL-33 exacerbates eosinophil-mediated airway inflammation. J Immunol. 2010;185(6):3472-3480. doi:10.4049/jimmunol.1000730

58. Kim DK, Jin HR, Eun KM, et al. The role of interleukin-33 in chronic rhinosinusitis. Thorax. 2017;72(7):635-645. doi:10.1136/ thoraxjnl-2016-208772

59. Liu T, Kanaoka Y, Barrett NA, et al. Aspirin-exacerbated respiratory disease involves a cysteinyl leukotriene-driven IL-33-mediated mast cell activation pathway. $J$ Immunol. 2015;195(8):3537-3545. doi:10.4049/jimmunol.1500905

60. Zhang L, Zhang Y, Gao Y, et al. Long-term outcomes of different endoscopic sinus surgery in recurrent chronic rhinosinusitis with nasal polyps and asthma. Rhinology. 2020;58(2):126-135. doi:10.4193/rhin19.184

61. Soler ZM, Jones R, Le P, et al. Sino-nasal outcome test-22 outcomes after sinus surgery: a systematic review and meta-analysis. Laryngoscope. 2018;128(3):581-592. doi:10.1002/lary.27008

62. Smith TL, Schlosser RJ, Mace JC, et al. Long-term outcomes of endoscopic sinus surgery in the management of adult chronic rhinosinusitis. Int Forum Allergy Rhinol. 2019;9(8):831-841. doi:10.1002/alr.22369

63. Gurrola J, Borish L. Chronic rhinosinusitis: endotypes, biomarkers, and treatment response. $J$ Allergy Clin Immunol. 2017;140 (6):1499-1508. doi:10.1016/j.jaci.2017.10.006

64. Tomassen P, Vandeplas G, Van Zele T, et al. Inflammatory endotypes of chronic rhinosinusitis based on cluster analysis of biomarkers. J Allergy Clin Immunol. 2016;137(5):1449-1456.e4. doi:10.1016/j. jaci.2015.12.1324

\section{Publish your work in this journal}

The Journal of Asthma and Allergy is an international, peer-reviewed open-access journal publishing original research, reports, editorials and commentaries on the following topics: Asthma; Pulmonary physiology; Asthma related clinical health; Clinical immunology and the immunological basis of disease; Pharmacological interventions and new therapies. The manuscript management system is completely online and includes a very quick and fair peer-review system, which is all easy to use. Visit http://www.dovepress.com/testimonials.php to read real quotes from published authors. 